\title{
CORRECTION
}

\section{Correction to: Implementation of peer learning conferences throughout a multi-site abdominal radiology practice}

\author{
Andrew W. Bowman ${ }^{1}$ (D) Nelly $\operatorname{Tan}^{2} \cdot$ Daniel A. Adamo ${ }^{3} \cdot$ Frederick Chen $^{2} \cdot$ Sudhakar K. Venkatesh ${ }^{3}$. \\ Deborah A. Baumgarten ${ }^{1}$
}

Published online: 11 June 2021

(c) Springer Science+Business Media, LLC, part of Springer Nature 2021

\section{Correction to: Abdominal Radiology https://doi.org/10.1007/s00261-021-03114-8}

The original version of this article unfortunately contained mistakes in Fig. 1B and Fig. 3. The correct figures are given below.

The original article has been corrected.

1 Department of Radiology, Mayo Clinic, 4500 San Pablo Rd, Jacksonville, FL 32224, USA

2 Department of Radiology, Mayo Clinic, 5777 Mayo Blvd, Phoenix, AZ 85054, USA

3 Department of Radiology, Mayo Clinic, 200 First St SW, Rochester, MN 55905, USA 


\section{b}

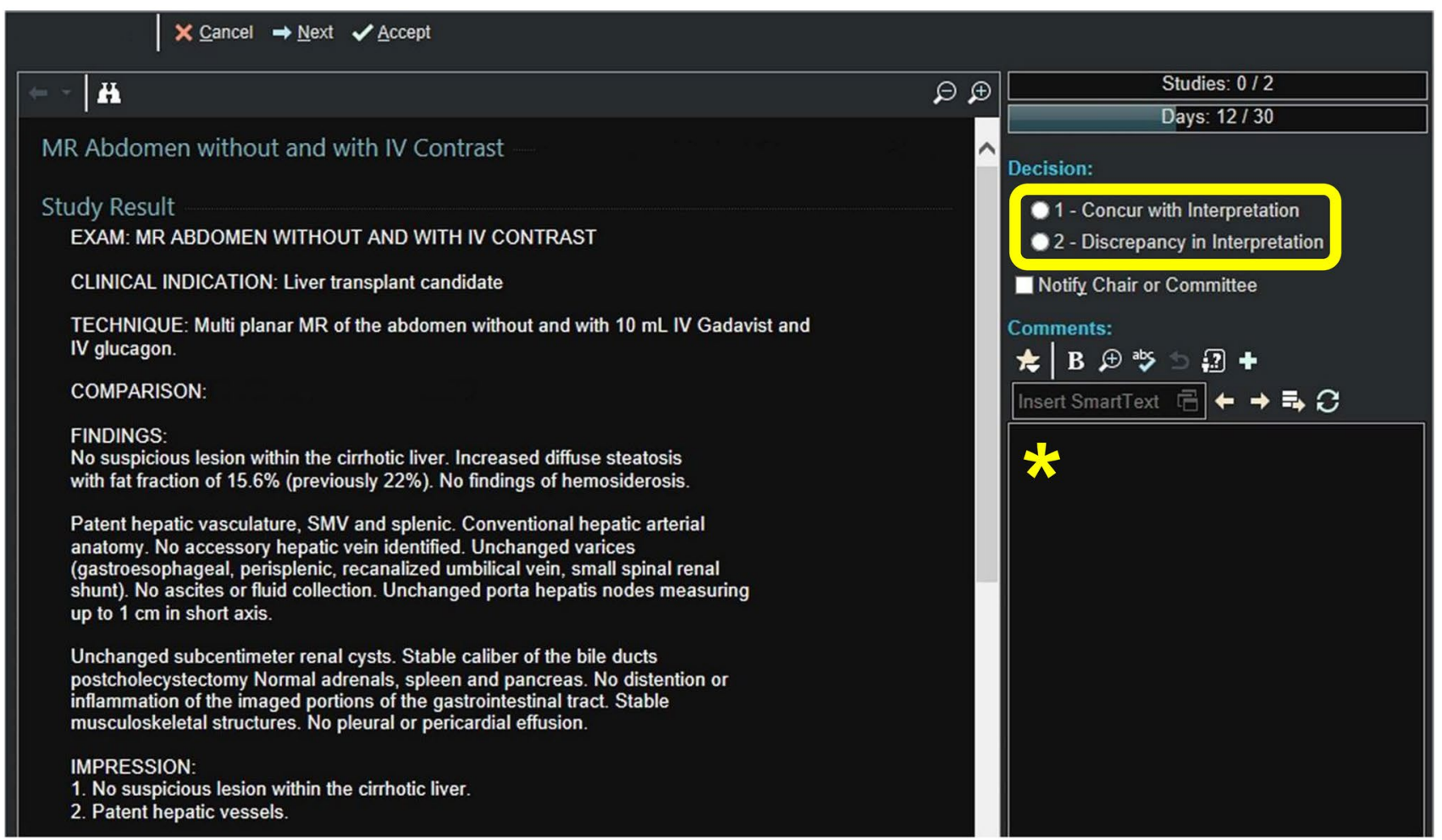

Fig. 1 Screenshot examples of our baseline Peer-Review process. a Screenshot of a portion of the Radiology dashboard from our electronic medical record (EMR) demonstrates the status of the monthly $\mathrm{PR}$ requirements. All radiologists are assigned to pools to review specific types of exams (in this particular example, body MR exams performed from the FL campus). When ready to peer review a case, the radiologist clicks on the "Review study" hyperlink shown in red text (arrow), which launches a case in our integrated imaging viewer (not shown) as well as a popup window with the anonymized report (b).
The radiologist reviews the imaging and report, then either concurs with the report or indicates a discrepancy in interpretation (yellow oval in b). When discrepancies are marked, there is a text box in the report window ( $*$ in $\mathbf{b}$ ) where the reason for the discrepancy can be explained. Cases marked as discrepant are automatically forwarded to the local quality chair for each site, who then reviews the case with the original interpreting radiologist to determine whether or not the discrepancy should be considered significant 
Fig. 3 Screenshot examples from our new process to submit a case for Peer Learning conferences. a Screenshot of our Radiology EMR with a case open (study images not shown). Our EMR was constructed with a tool for saving interesting cases for any reason (yellow oval). Once selected, the Interesting Case tool launches a menu to further characterize the case in question (b). When "Teaching Case" is selected (highlighted in blue in $\mathbf{b}$ ), a free text window opens where the reason for the case being interesting can be entered. We include the phrase "peer learning" in the comments box and explain why the case is a learning opportunity or a great call. We can then filter through all saved cases in this system using the phrase "peer learning" to retrieve all the cases submitted in this fashion a

MR Abdomen and Liver Elactnnram without and with IV Contrast, Exam date:

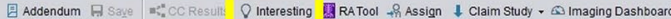

Study Background FeedbackLLOS CTRM Miscellaneous

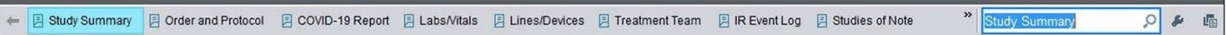

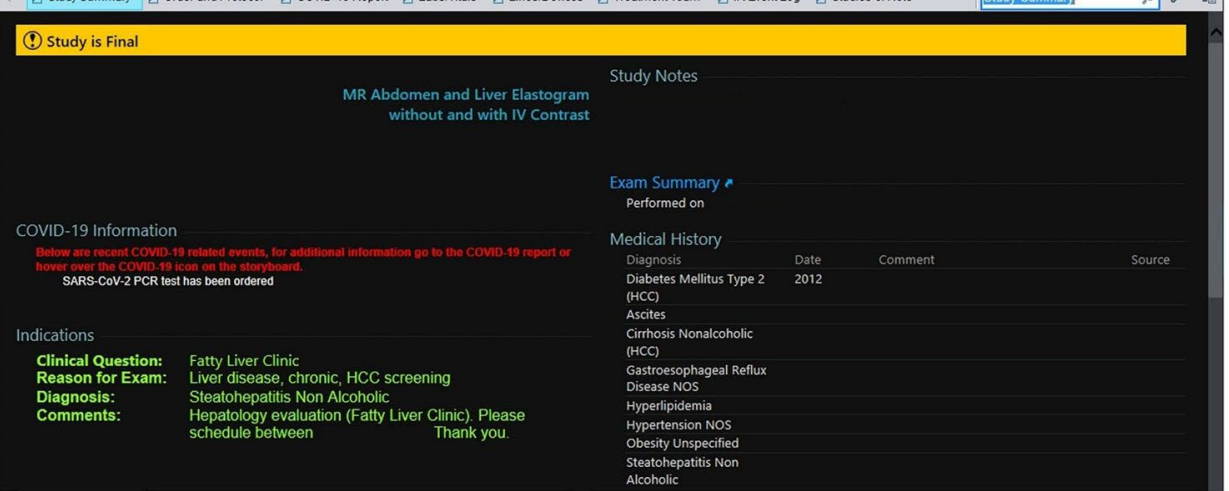

b

MR Abdomen and Liver Elastogram without and with IV Contrast, Exam date: Study Background FeedbackLoS CTRM Miscellaneous - E Study Summary 目 Order and Protocol 目 CoviD-19R (C) Study is Final
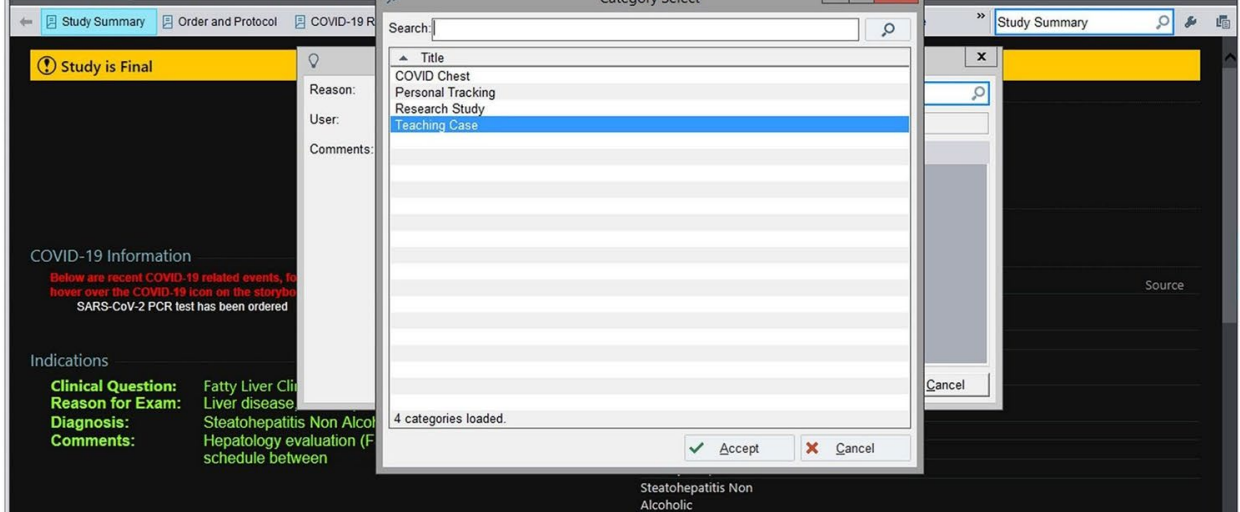

C

MR Abdomen and Liver Elastogram without and with IV Contrast, Exam date:

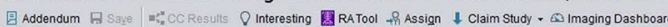
Study Background Feedbacklos CTRM Miscellaneous

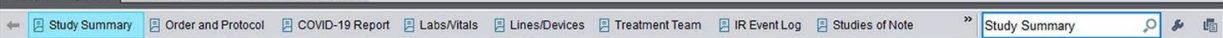

(1) Study is Fin

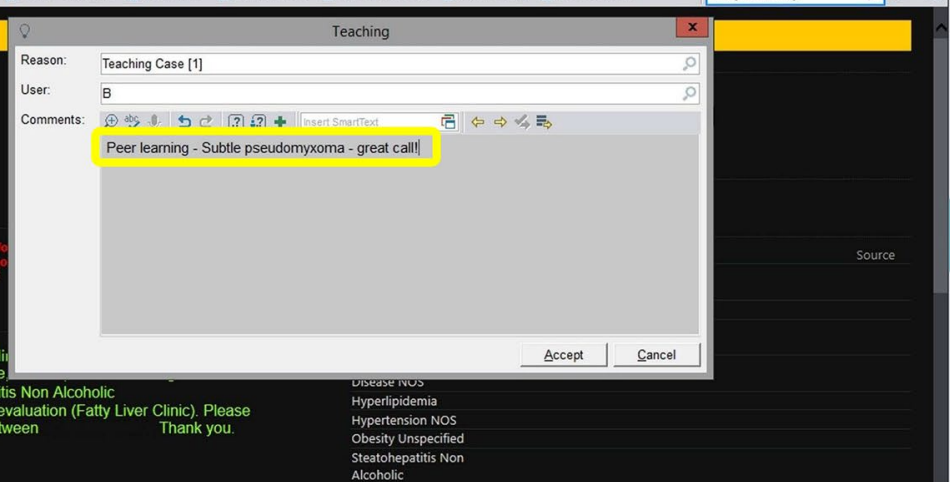

Publisher's Note Springer Nature remains neutral with regard to jurisdictional claims in published maps and institutional affiliations. 